\title{
Effects of Pringle maneuver on systemic hemodynamics during liver resection surgery under thoracic epidural anaesthesia. Role of dynamic arterial elastance (Eadyn).
}

Authors: Varela J.A, Perez Peña J, Lisbona C, Blanco J, De Miguel A, Olmedilla L.

Institute: Gregorio Marañon General Hospital, Deptarment of Anaesthesiology \& Intensive Care, Madrid, Spain.

\section{Background and Goal of Study}

Occlusion of hepatic blood flow by Pringle maneuver (PM) is used in liver surgery to minimize blood loss. The aim of this study was to describe the hemodynamic changes during PM and the use of dynamic arterial elastance (Eadyn) as a measure of vascular tone to predict this response during liver resection in patients under epidural anaesthesia.

\section{Material and methods}

61 patients who underwent liver open resection with PM and combined general and thoracic epidural anaesthesia were included. Major hepatectomy was performed in 21 cases. There were 47 men and 14 women, ASA I-III, with a mean age of 65 years (39-84). Mean PM duration was 23+/16 min. Hemodynamic parameters were monitored with a transpulmonary thermodilution system (PiCCO, Pulsion Medical Systems, Germany): heart rate (HR), mean arterial pressure (MAP), central venous pressure (CVP), cardiac index $(\mathrm{Cl})$, stroke volume variation (SVV), pulse pressure variation (PPV), global end diastolic index (GEDI), cardiac function index (CFI), left heart contractility $(\mathrm{dPmx})$, systemic vascular resistance index (SVRI) and Eadyn (PPV/SVV). They were recorded at 3 time points: $5 \mathrm{~min}$ before (T1) and 5 min after clamping (T2) and 5 min after unclamping (T3). Statistical analysis: Student's T test. A p-value $<0.05$ was considered statistically significant.

\section{Results}

PM produced a mild reduction in venous return (CVP $-11 \%$, SVV and PPV $-20 \%$ ) and in $\mathrm{Cl}(-13 \%)$. Decreased in contractility (dPmx $-12 \%, \mathrm{CFI}-9 \%)$ was compensated by an increase in vascular tone (SVRI and Eadyn $+8 \%$ ) in order to keep MAP unchanged despite the sympathetic epidural block

At T3 HR, MAP, Cl, CFI and dPmx significantly increased above T1 values but SVRI and Eadyn fell under their T1 values. SVV and PPV values were similar to T1 ones.

\begin{tabular}{|c|c|c|c|}
\hline & T1 (pre- Pringle) & T2 (Pringle) & T3 (post- Pringle) \\
\hline CVP & $9 \pm 3$ & $8 \pm 4$ & $8 \pm 4$ \\
\hline SVV & $15 \pm 5$ & $18 \pm 6$ & $15 \pm 6$ \\
\hline PPV & $14 \pm 5$ & $17 \pm 6$ & $13 \pm 5$ \\
\hline $\mathrm{CFI}$ & $3,5 \pm 1$ & $3,2 \pm 0,9$ & $3,8 \pm 0,9$ \\
\hline $\mathrm{dPmx}$ & $896 \pm 402$ & $790 \pm 387$ & $1023 \pm 412$ \\
\hline SVRI & $2415 \pm 739$ & $2596 \pm 715$ & $2271 \pm 611$ \\
\hline Eadyn & $0,92 \pm 0,21$ & $0,99 \pm 0,25$ & $0,89 \pm 0,24$ \\
\hline MAP & $71 \pm 13$ & $68 \pm 14$ & $75 \pm 13$ \\
\hline
\end{tabular}

The group of patients with low vascular tone (Eadyn $<0.8$ ) at T1 underwent a greater decrease in MAP between T1 and T3 $(-7 \pm 13$ vs $+5 \pm 15 p$ 0.01), and had lower MAP (68 \pm 11 vs $76 \pm 13$ p 0.04 ) at T3 than those with Eadyn $>0.8$.

Patients with Eadyn $<0.8$ showed higher $\mathrm{dPmx}$ at $\mathrm{T} 1$ $(1198 \pm 566$ vs $797 \pm 277)$ and T2 (975 $\pm 548 v s \quad 723 \pm 298)$ and

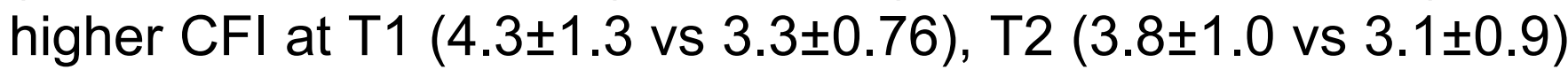
and T3 (4.3 \pm 1.0 vs $3.7 \pm 0.9)$ compared to the group with Eadyn $>0.8$ ( $p<0.03$ ). SVRI measured before, during and after PM was lower (not significant) in the Eadyn $<0.8$ group. The rest of hemodynamic parameters showed no changes to note.

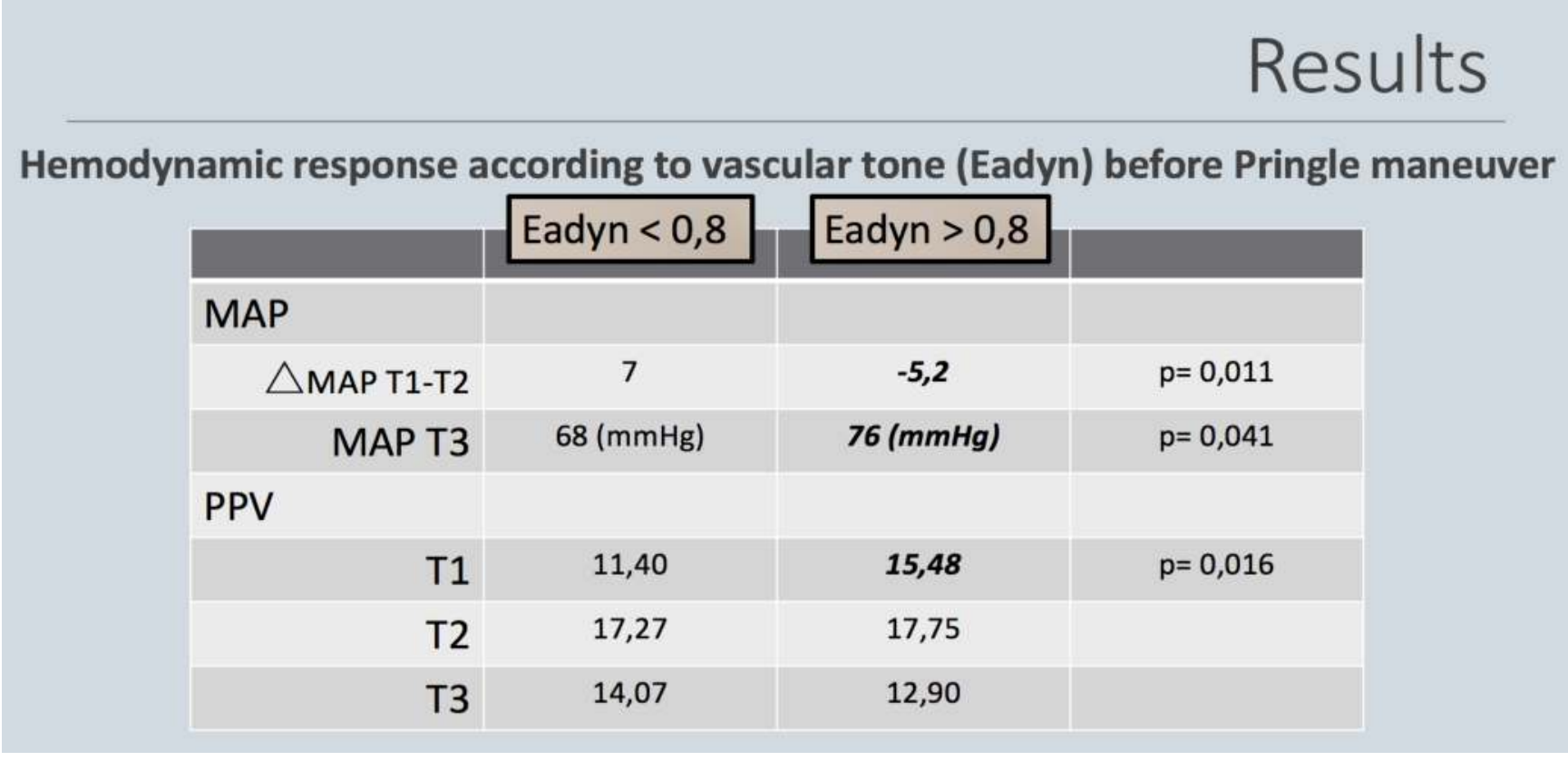

Results

Hemodynamic response according to vascular tone (Eadyn) before Pringle maneuver

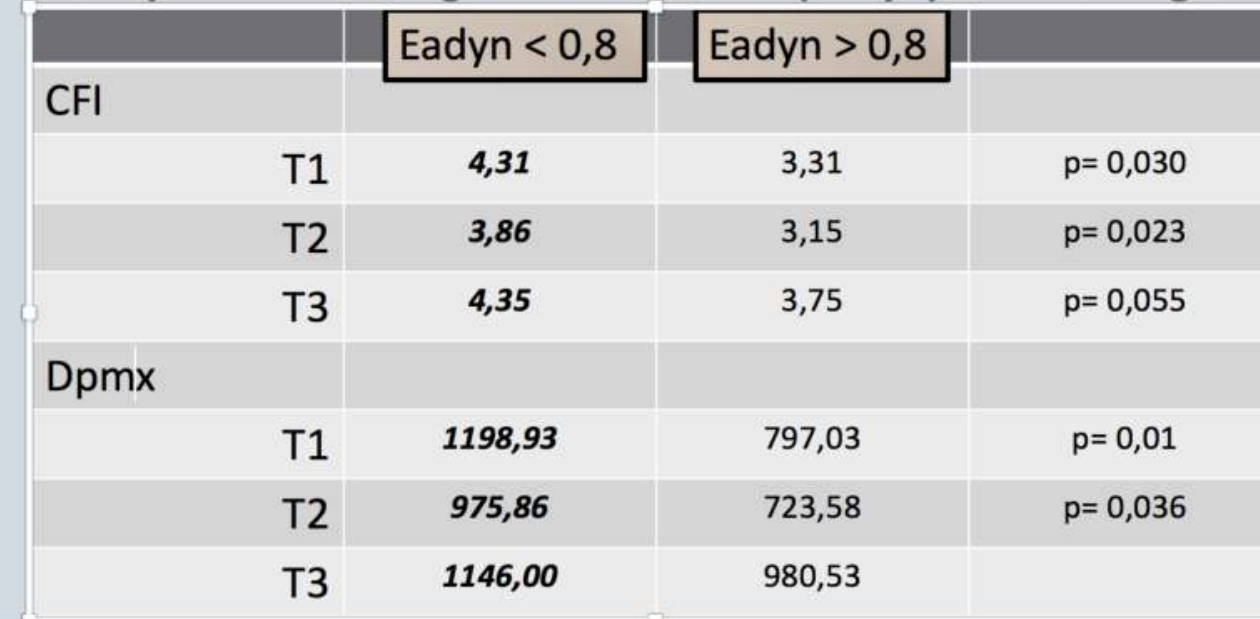

\section{Conclusions}

- Hemodynamic changes induced by PM in patients with thoracic epidural block are compensated with a reflex increase in vascular tone.

- Patients with lower vascular tone before PM (Eadyn <0.8) show higher MAP reduction despite the contractility increase.

\section{References}

1.- Dynamic arterial elastance predicts mean arterial pressure decrease associated with decreasing norepinephrine dosage in septic shock. Guinot et al. Critical Care (2015) 19:14

2.- Dynamic arterial elastance as a predictor of arterial pressure response to fluid administration: a validation study. García et al. Critical Care 2014, 18:626 